\title{
VERITAS Observations of the Galactic Center
}

\author{
Amanda Weinstein for the VERITAS Collaboration* \\ Iowa State University \\ E-mail: amandajweiastate.edu
}

Weakly-interacting massive particles (WIMPS) are among the wide range of candidates for the dark matter (DM) that dominates the mass content of the universe. In some scenarios these WIMPs are self-annihilating. In regions of high dark matter density (such as our own Galactic Center) this self-annihilation is expected to produce a characteristic gamma-ray radiation signature that cuts off at the WIMP mass. Observations of these regions of high dark matter density with very-high-energy (VHE) gamma-ray telescopes can constrain a unique phase space of heavy WIMP masses. However, in the case of the Galactic Center gamma-ray emission arising from more conventional astrophysical sources complicates the interpretation of such observations. We provide an update on deep observations of the Galactic Center with VERITAS, an array of atmospheric Cherenkov telescopes sensitive to VHE gamma rays with energies between $85 \mathrm{GeV}$ and $30 \mathrm{TeV}$.

38th International Conference on High Energy Physics 3-10 August 2016

Chicago, USA

${ }^{*}$ Speaker. 


\section{Motivation}

Weakly-interacting massive particles (WIMPS) are among the wide range of candidates for the dark matter (DM) that dominates the mass content of the universe. In some scenarios (e.g. neutralinos) these WIMPs are self-annihilating. In regions of high dark matter density this selfannihilation is expected to produce a characteristic gamma-ray radiation signature that cuts off at the WIMP mass. Observations of these regions of high dark matter density with very-high-energy (VHE) gamma-ray telescopes can constrain a unique phase space of heavy WIMP masses. Such regions of high dark matter density may be found at the centers of galaxies, in galaxy clusters, and in substructures of the Galactic halo. Dwarf spheroidal galaxies, with their high mass-to-light ratio and corresponding lack of gamma-ray emission from conventional astrophysical sources, would provide the most easily interpreted gamma-ray signature of dark matter annihilation (or decay). However, the Galactic Center is generally expected to provide the strongest absolute signal [6].

Any indirect dark matter search using the Galactic Center must contend with an intense foreground of gamma-ray emission from a variety of sources. Some of these sources, such as the well-known source Sgr A*, are extremely bright but compact, while others, such as the diffuse emission produced by cosmic rays interacting with molecular clouds, are on a large angular scale. We provide an update on deep observations of the Galactic Center with VERITAS, an array of atmospheric Cherenkov telescopes sensitive to VHE gamma rays with energies between $85 \mathrm{GeV}$ and $30 \mathrm{TeV}$. We consider both new results related to the conventional astrophysical above $2 \mathrm{TeV}$ and prospects for future indirect dark matter searches with these data.

\section{VERITAS}

The Very Energetic Radiation Imaging Telescope Array System (VERITAS) is an array of four 12-m imaging atmospheric Cherenkov telescopes (IACTs) located at the Fred Lawrence Whipple Observatory in southern Arizona. The array has operated continuously since its commissioning in 2007. Under normal operating conditions, VERITAS is sensitive to a point source with $1 \% \mathrm{Crab}$ nebula flux in 25 hours of observations. The energy resolution of the instrument (under these same conditions) is approximately $15 \%$ at $1 \mathrm{TeV}$. The angular resolution varies with energy but it typically less than $0.1^{\circ}$ above $1 \mathrm{TeV}$. However, as we note below, the instrument performance changes when observing at zenith angles greater than $60^{\circ}$.

Between 2010 and 2014, VERITAS accumulated approximately 85 hours (effective) of qualityselected observations of the portion of the Galactic Center Ridge immediately surrounding Sgr A*. These observations were taken at elevations between $25^{\circ}$ and $30^{\circ}$ elevation (as VERITAS is in the Northern Hemisphere, the Galactic Center never transits any higher than this). At these elevations, the parallactic displacements between shower images in two or more telescopes may be small and the information they provide about the shower effectively degenerate. Since the method used to reconstruct information about the primary gamma ray relies on these shower images providing independent constraints, this leads to a significant degradation in angular resolution. For the measurements described here, a special "Displacement" [8,4] method was used to compensate for this degradation. Large zenith angle observations also result in a higher effective energy threshold for the instrument, since the brightness of any shower image is attentuated by the larger atmospheric 
overburden. However, that same effect increases the effective area of the instrument for energies above a few $\mathrm{TeV}$. This confers a statistical advantage when measuring the gamma-ray spectrum of a source at multi- $\mathrm{TeV}$ energies.

\section{SGR $A^{*}($ VER J1745-290) and G0.9+1.0}

VERITAS detects a total of 735 excess gamma-ray events from a source seen at the position of Sgr A*, also referred to as VER J1745-290 [5]. The significance of the detection is greater than 25 standard deviations $(\sigma)$, an increase of over seven standard deviations from that reported in previous work using a smaller data sample [4]. The increased statistics permit an improved determination of the position for VER J1745-290 of $l=359.94^{\circ} \pm 0.002_{\text {stat }}^{\circ} \pm 0.013_{\text {sys }}^{\circ}$ and $b=$ $-0.053^{\circ} \pm 0.002_{\text {stat }}^{\circ} \pm 0.013_{\text {sys }}^{\circ}$ [5]. As shown in Figure 1, the position of the radio source [10], the position of the gamma-ray source as reported by the H.E.S.S. observatory, and the updated VERITAS position are all in good agreement with one another. Figure 1 shows the spectrum of Sgr $A^{*}$ from $2 \mathrm{TeV}$ to $30 \mathrm{TeV}$, as derived from a circular region $0.13^{\circ}$ in radius centered on the nominal VERITAS position. When combined with the spectral points above $200 \mathrm{GeV}$ provided by H.E.S.S., the combined spectrum fits well to both a power law spectrum with an exponential cutoff and a smoothly broken power law. A pure power law is disfavored $\left(\chi^{2} /\right.$ ndf of $148 / 32$ for a pure power law, vs $\chi^{2} /$ ndf $\sim 1.1$ for the alternatives). Since large zenith angle observations boost the effective area at high energies, the VERITAS observations provide improved statistics at high energies. The VERITAS systematic error is increased by the large zenith angle to $\sim 40 \%$ on both the energy scale and flux normalization and dominates the systematic errors for the combined spectrum.

It should be noted that the VERITAS spectrum of Sgr A* includes contamination from the diffuse emission. However, given statistical and systematic errors, the resulting spectrum is consistent with alternative analyses of H.E.S.S. data that explicitly subtract a model of this emission [11]. In particular, the cutoff energy derived by fitting the combined H.E.S.S.-VERITAS spectrum with a power law with an exponential cutoff $(12.1 \pm 1.6 \mathrm{TeV})$ is consistent within errors with a cutoff derived in [11].

The second bright gamma-ray point source corresponds to a radio shell-type supernova remnant $(\mathrm{G} 0.9+1.0)$ surrounding a radio pulsar wind nebula $(\mathrm{PWN})$ [7]. It was first established as a gamma-ray source above $200 \mathrm{GeV}$ by H.E.S.S.. The VERITAS source agrees well in position with both the H.E.S.S. location and that of the radio PWN. The joint VERITAS and H.E.S.S. spectrum fits well to a pure power law, with no significant indication of a spectral break out to $20 \mathrm{TeV}$. The combined gamma-ray picture is consistent with emission arising from a TeV PWN that corresponds to the radio PWN, an interpretation bolstered by the lack of hard X-ray emission from the SNR shell.

\section{Galactic diffuse emission}

After masking out the two bright point sources Sgr A* and SNR G0.9+0.1, VERITAS observes the complex diffuse emission along the Galactic Center Ridge as shown in Figure 2 [5]. The morphology of the emission is broadly consistent with (but not identical to) the morphology of the 

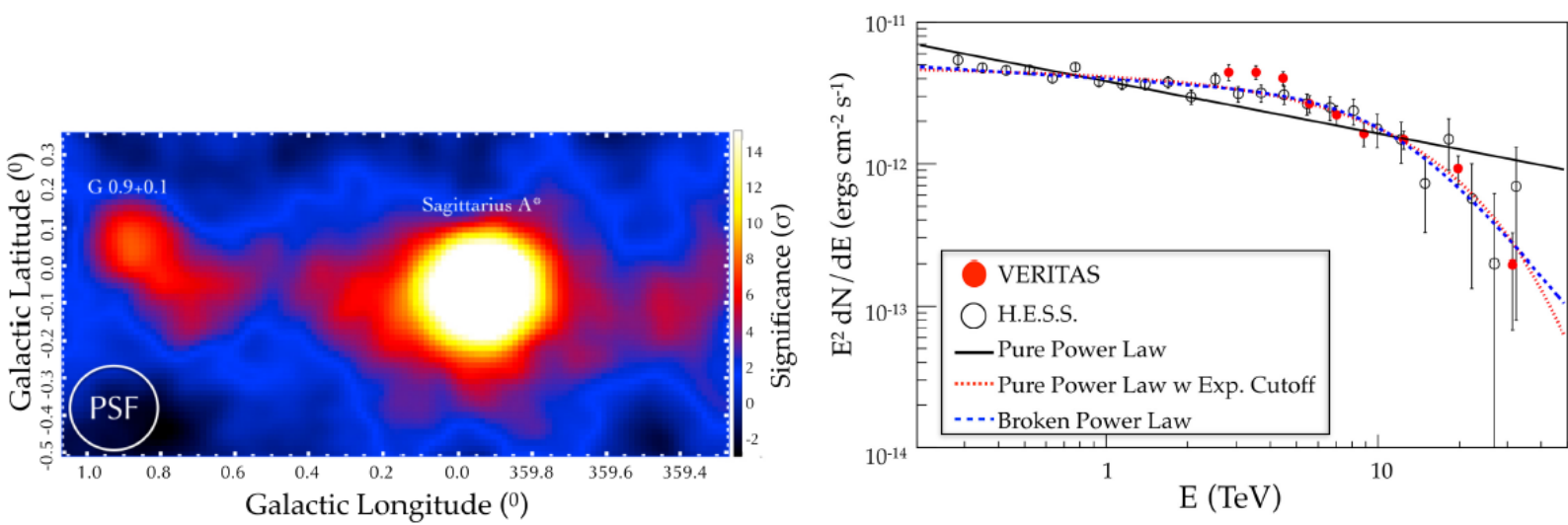

Figure 1: Left: VERITAS $>2 \mathrm{TeV}$ gamma-ray excess map of the Galactic Center ridge, showing the two bright point sources, Sgr A* and SNR G0.9+0.1, as well as the residual diffuse emission. The map has been smoothed with the energy-weighted VERITAS PSF. The color-scale has been truncated (saturating the emission from Sgr A* in order to improve contrast for dimmer features.

emission above $300 \mathrm{GeV}$ extracted from H.E.S.S. observations [3]. The overall similarity between the two maps and to the distribution of molecular material suggests that the diffuse emission seen by both instruments arises from the decay of $\pi^{0}$ s produced by the interaction of cosmic rays with $H I$ and $H I I$ regions $[1,5]$.

The most significant feature of the diffuse emission as seen by VERITAS is a local enhancement adjacent to Sgr A*, dubbed VER J1746-289. The excess is centered at $l=0.055^{\circ} \pm 0.01_{\text {stat }}^{\circ} \pm$ $0.013_{\text {sys }}^{\circ}, b=-0.148^{\circ} \pm 0.01_{\text {stat }}^{\circ} \pm 0.013_{\text {sys. }}^{\circ}$. It is well-described by an asymmetric two-dimensional Gaussian with $\sigma_{l}=0.08^{\circ}, \sigma_{b}=0.03^{\circ}$, rotated by $-15.4^{\circ}$ with respect to Galactic Latitude. This feature does not appear to be sensitive to the size of the region used to mask out Sgr A*, which argues against its being an artifact of the source subtraction. This structure coincides with a radio arc observed at $20 \mathrm{~cm} \mathrm{[12]} \mathrm{and} \mathrm{a} \mathrm{gamma-ray} \mathrm{source} \mathrm{from} \mathrm{the} \mathrm{Fermi-LAT} \mathrm{3FGL} \mathrm{catalog.} \mathrm{VER}$ J1746-289 also coincides with a similar enhancement in the $>200 \mathrm{GeV}$ diffuse emission observed by H.E.S.S., although the peaks of the emission above $200 \mathrm{GeV}$ and $2 \mathrm{TeV}$ are slightly offset. H.E.S.S. has established this excess as arising from a single point source [9], likely a pulsar wind nebula. VER J1746-289 is likely a combination of emission from this source and an enhancement in the diffuse emission above $\mathrm{TeV}$.

There is also a weaker enhancement near the giant molecular cloud complex Sgr B2, which (after accounting for trials factors) has a significance of $4.1 \sigma$, below the $5 \sigma$ threshold used to a claim a source detection by VERITAS.

\section{Prospects for indirect dark matter searches at the GC}

VERITAS large zenith angle observations of the Galactic Center allow indirect dark matter searches that target large WIMP masses. In particular, searches for gamma-rays arising from selfannihilation of neutralino dark matter have been considered. Figure 3 shows the region surrounding

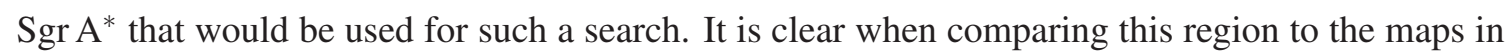




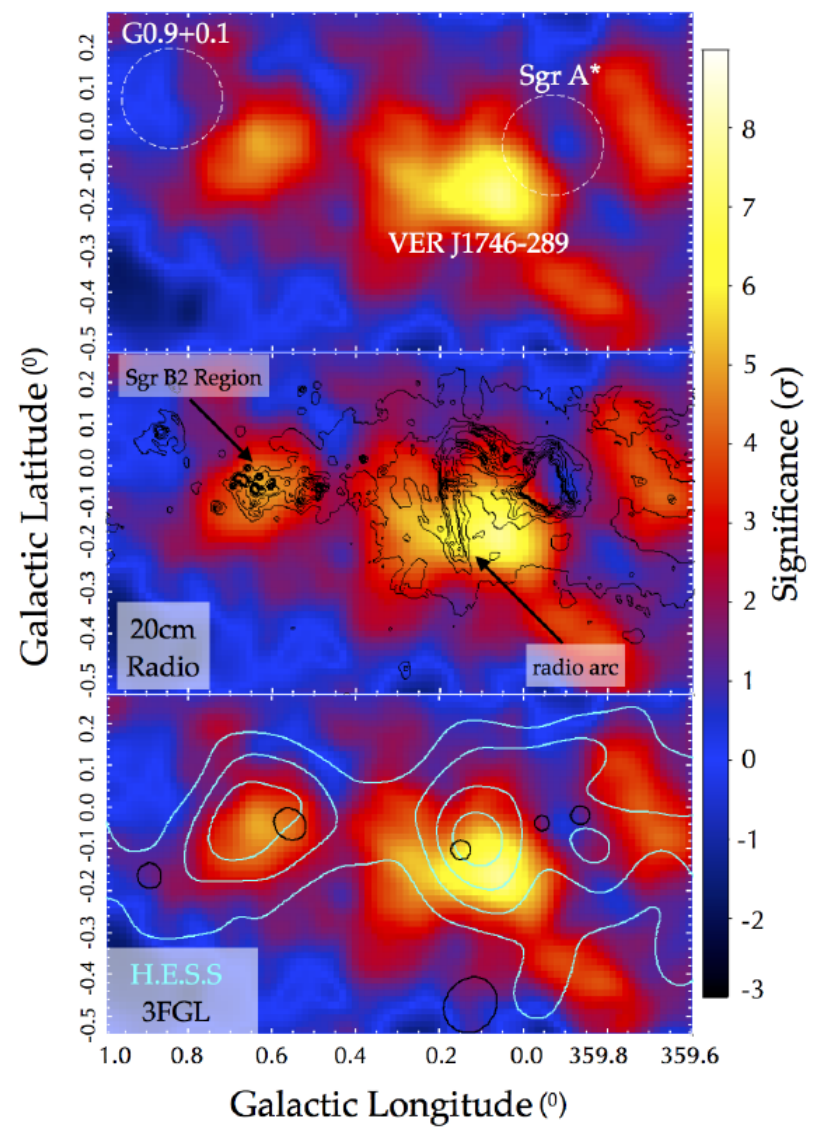

Figure 2: Maps of the VERITAS diffuse emission above $2 \mathrm{TeV}$ around the Galactic Center Ridge, after masking out emission from Sgr A* and SNR G0.9+0.1 (locations indicated in the top panel). Maps show the number of standard deviations above background for the gamma-ray emission at each position and are smoothed by the VERITAS PSF. Also shown are the location of VERITAS source VER J1746-289 (top panel); VLA 20cm radio contours from [12] (middle panel), and H.E.S.S. 275, 300, 325, and 350 excess event contours [3] and Fermi-LAT 3FGL [2] sources (bottom panel).

figure 2 that a more rigorous method of subtracting out both the bright point sources and diffuse emission components will be required. Such approaches are currently under investigation.

Figure 3 shows a preliminary projection of the sensitivity in terms of thermally-averaged neutralino cross-section vs. neutralino mass. For the purposes of simplification several different curves corresponding to different decay channels are shown, each assuming that $100 \%$ of neutralinos decay into that channel. Updated estimates, taking into account the actual amount of accumulated VERITAS data on the Galactic Center, as well as improved modeling of the dark matter distribution, will be forthcoming.

\section{Acknowledgments}

This research is supported by grants from the U.S. Department of Energy Office of Science, the U.S. National Science Foundation and the Smithsonian Institution, by NSERC in Canada, by 

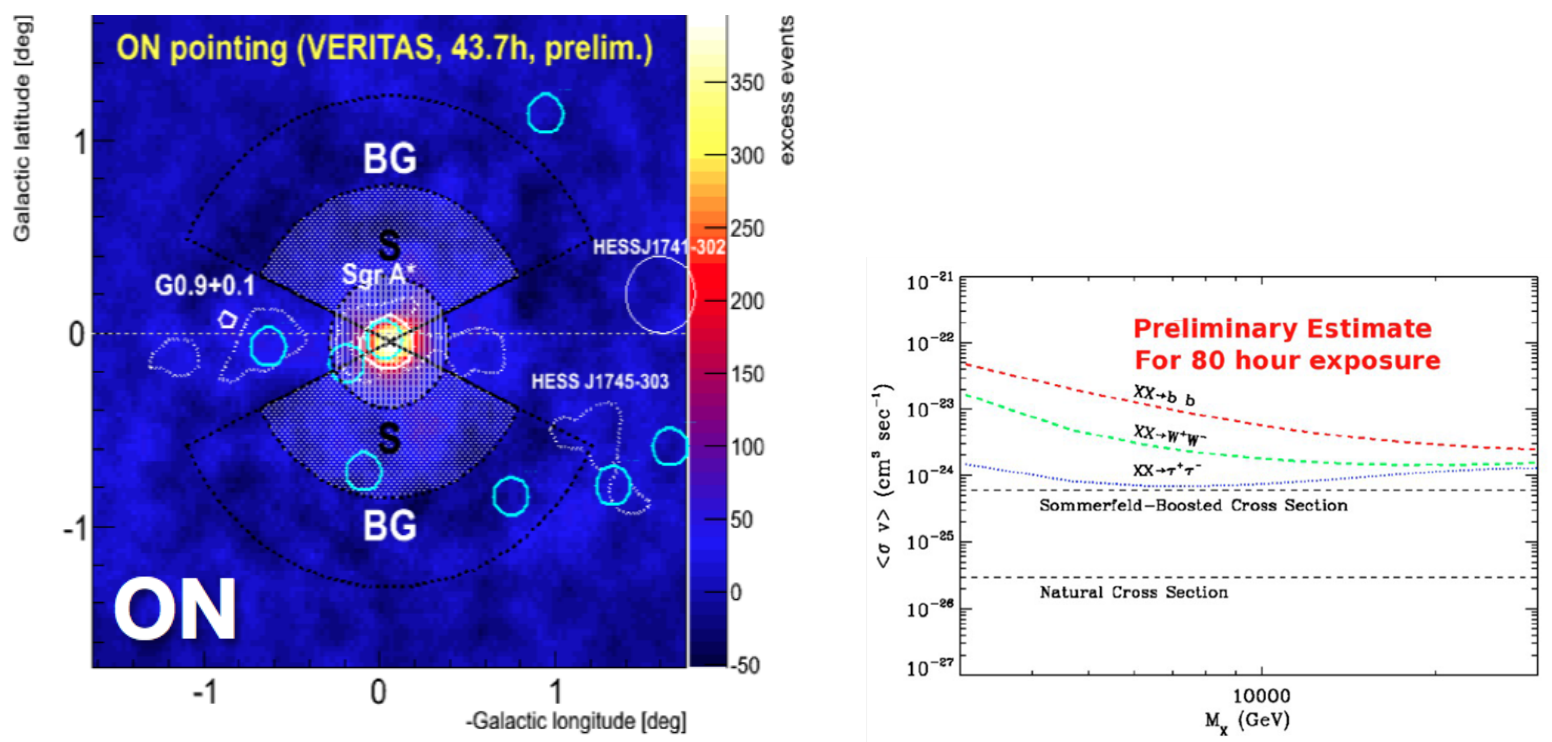

Figure 3: Left: Map illustrating the regions that will be used to search for gamma-ray emission from dark matter self-annihilation (marked with "S") and regions used to estimate backgorund ("BG"). Right: Preliminary projection of indirect dark matter search sensitivity with 80 hours of VERITAS data on the Galactic Center, in terms of thermally-averaged neutralino cross section vs. neutralino mass.

Science Foundation Ireland (SFI 10/RFP/AST2748). We acknowledge the excellent work of the technical support staff at the Fred Lawrence Whipple Observatory and at the collaborating institutions in the construction and operation of the instrument. The VERITAS Collaboration is grateful to Trevor Weekes for his seminal contributions and leadership in the field of VHE gamma-ray astrophysics, which made this study possible.

\section{References}

[1] Abramowski, A., Aharonian, F., Ait Benkhali, F., et al. 2014, Phys. Rev. D , 90, 122007

[2] Acero, F., Ackermann, M., Ajello, M., et al. 2015, ApJS , 218, 23

[3] Aharonian, F., Akhperjanian, A. G., Bazer-Bachi, A. R., et al. 2006, Nature , 439, 695

[4] Archer, A., Barnacka, A., Beilicke, M., et al. 2014, ApJ , 790, 149

[5] Archer, A., Benbow, W., Bird, R., et al. 2016, ApJ , 821, 129

[6] Gaskins, J. M. 2016, Contemporary Physics, 57, 496

[7] Helfand, D. J., \& Becker, R. H. 1987, ApJ , 314, 203

[8] Kosack, K., Badran, H. M., Bond, I. H., et al. 2004, ApJ , 608, L97

[9] Lemiere, A., Terrier, R., Jouvin, L., et al. 2015, arXiv:1510.04518

[10] Petrov, L., Kovalev, Y. Y., Fomalont, E. B., \& Gordon, D. 2011, AJ , 142, 35

[11] Viana, A. and Moulin, E 2014, BrJPh, 44, 415

[12] Yusef-Zadeh, F., Hewitt, J. W., \& Cotton, W. 2004, ApJS , 155, 421 